\title{
Formation of W/O Microemulsion Based on Natural Glycolipid Biosurfactant, Mannosylerythritol Lipid-A
}

\author{
Wannasiri Worakitkanchanakul ${ }^{1}$, Tomohiro Imura ${ }^{2}$, Tomotake Morita ${ }^{2}$, \\ Tokuma Fukuoka ${ }^{2}$, Hideki Sakai ${ }^{1,3}$, Masahiko Abe ${ }^{1,3}$, Ratana Rujiravanit ${ }^{4}$, \\ Sumaeth Chavadej ${ }^{4}$ and Dai Kitamoto ${ }^{2 *}$ \\ ${ }^{1}$ Faculty of Science and Technology, Tokyo University of Science (2641 Yamazaki, Noda, Chiba 278-8510, JAPAN) \\ ${ }^{2}$ Research Institute for Innovation in Sustainable Chemistry, National Institute of Advanced Industrial Science and Technology (AIST) \\ (Tsukuba Central 5-2, Higashi 1-1, Tsukuba, Ibaraki 305-8565, JAPAN) \\ ${ }^{3}$ Institute of Colloid and Interfacial Science, Tokyo University of Science (1-3 Kagurazaka, Shinjyuku, Tokyo 162-8601, JAPAN) \\ ${ }^{4}$ The Petroleum and Petrochemical College, Chulalongkorn University (Soi Chula 12, Phya Thai Rd., Pathumwan, Bangkok 10330, \\ THAILAND)
}

\begin{abstract}
Mannosylerythritol lipid-A (MEL-A) is a glycolipid biosurfactant abundantly produced from soybean oil by microorganisms at a yield of up to $100 \mathrm{~g} / \mathrm{L}$. In this study, the formation of water-in-oil (W/O) microemulsion based on the single component of MEL-A was confirmed using dynamic light scattering (DLS) and freeze fracture electron microscopy (FF-EM). DLS and FF-EM measurements revealed that the diameter of the microemulsion increases with an increase in water-to-surfactant mole ratio $\left(W_{0}\right)$ ranging from 20 to $60 \mathrm{~nm}$, and the maximum $W_{0}$ value was found to be 20 , which is as high as that of soybean lecithin. Glycolipid biosurfactant has a great potential for the formation of W/O microemulsion without using any cosurfactants.
\end{abstract}

Key words: glycolipid biosurfactant, mannosylerythritol lipid-A, W/O microemulsion, freeze fracture electron microscopy (FF-EM), dynamic light scattering (DLS)

\section{INTRODUCTION}

Microemulsions are thermodynamically stable, isotropically homogeneous transparent systems containing substantial amounts of two immiscible liquids (i.e. water and oil) stabilized by appropriate surfactants ${ }^{1-4)}$. They are formed when their interfacial tension is extremely low $\left(<10^{-2} \mathrm{mN} / \mathrm{m}\right)^{2,3)}$, and are generally classified into three types water-in-oil (W/O), oil-in-water $(\mathrm{O} / \mathrm{W})$, and bicontinuous microemulsions depending on the spontaneous curvature $\left(\mathrm{H}_{0}\right)$ of the surfactant monolayers at the interface ${ }^{4)}$. Recently, W/O microemulsion has attracted attention in various fields of application, including dry cleaning ${ }^{1)}$, cosmetics ${ }^{5}$, and bio-diesels ${ }^{6}$. One of the interesting advantages of microemulsions is that microemulsion-based fuels contribute to decreasing the emission rate of nitrogen oxides $\left(\mathrm{NO}_{\mathrm{x}}\right)$ and carbon monoxide $(\mathrm{CO})$ with improved fuel economy $y^{3)}$. However, the formation of microemulsions usually requires the use of surfactant mixtures with salt or alcohol ${ }^{2,47}$, and only a few surfactants such as sodium bis(2-ethylhexyl) sulfosuccinate $(\mathrm{AOT})^{8-10)}$ or soybean lecithin ${ }^{11)}$ are known to effectively form W/O microemulsion without addition of any cosurfactants: this has limited widespread application of the microemulsion technology.

Biosurfactants are "natural" surfactants which are abundantly produced from natural resources such as vegetable oils by microorganisms ${ }^{12}$. They have many advantages over "synthetic" surfactants, including their mild production conditions and higher environmental compatibility ${ }^{12}$. We have previously reported that mannosylerythritol lipidA (MEL-A), which is abundantly produced by yeast strains of Pseudozyma antarctica from soybean oil at a yield of up to $100 \mathrm{~g} / \mathrm{L}$, gives not only an excellent interfacial (water $/ n$ -

\footnotetext{
*Correspondence to: Dai Kitamoto, Research Institute for Innovation in Sustainable Chemistry, National Institute of Advanced Industrial Science and Technology (AIST), Tsukuba Central 5-2, Higashi 1-1, Tsukuba, Ibaraki 305-8565, JAPAN

E-mail: dai-kitamoto@aist.go.jp
}

Accepted October 24, 2007 (received for review August 3, 2007)

Journal of Oleo Science ISSN 1345-8957 print / ISSN 1347-3352 online

http://jos.jstage.jst.go.jp/en/ 


\section{W. Worakitkanchanakul, T. Imura, T. Morita et al.}

tetradecane) tension lowering action ${ }^{12)}$ but also a variety of self-assembled structures including sponge $\left(\mathrm{L}_{3}\right)^{13-15)}$ and reverse bicontinuous cubic $\left(\mathrm{V}_{2}\right)^{14,15)}$ phases in water, indicating that MEL-A assemblies have a negative spontaneous curvature $^{13,14)}$. These results imply that MEL-A would be useful for the formation of W/O microemulsion.

In this study, we investigate the phase behavior of a ternary MEL-A/water/n-decane system using dynamic light scattering (DLS) and freeze fracture electron microscopy (FF-EM), and report for the first time W/O microemulsion formation stabilized by natural glycolipid biosurfactant without adding any cosurfactants.

\section{EXPERIMENTAL}

\subsection{Materials and sample preparation}

4-O-(4',6'-Di- $O$-acetyl-2',3'-di- $O$-alkanoyl- $\beta$-D-mannopyranosyl)-D-erythritol (MEL-A) was obtained according to the following procedure ${ }^{15)}$ : a seed culture $(1.5 \mathrm{~mL})$ of the yeast strain Pseudozyma antarctica was transferred to $300 \mathrm{~mL}$ Erlenmeyer flasks containing $30 \mathrm{~mL}$ of a fermentation medium $\left[4 \%(\mathrm{v} / \mathrm{v})\right.$ soy bean oil, $0.2 \% \mathrm{NaNO}_{3}, 0.02 \% \mathrm{MgSO}_{4}$. $7 \mathrm{H}_{2} \mathrm{O}, 0.02 \% \mathrm{KH}_{2} \mathrm{PO}_{4}, 0.1 \%$ yeast extract, distilled water], and incubated on a rotatory shaker $(220 \mathrm{rpm})$ at $30^{\circ} \mathrm{C}$ for one week. The culture broth $(30 \mathrm{~mL})$ was then extracted twice with $30 \mathrm{~mL}$ portions of ethyl acetate. The layers were separated, and the organic layer was washed with brine and concentrated under reduced pressure. The resulting yellow oil (555 mg) was dissolved in chloroform (5 $\mathrm{mL})$ and placed on a column $(3 \times 40 \mathrm{~cm})$ of silica gel $(50 \mathrm{~g})$. The crude MELs were then subjected to chromatography with a close-gradient elution of chloroform/acetone (10:0 to 5:5). Each fraction was collected and again subjected to chromatography as described above to give MEL-A. MEL$\mathrm{A}$ has the fatty acid composition of $18 \% \mathrm{C} 8,71 \% \mathrm{C} 10$, and $11 \%$ C12. The purity of MEL-A (>99\%) was confirmed by high performance liquid chromatography. $n$-Decane (Wako Pure Chemical Industries, Ltd.) was selected as an oil phase and used as received. An oil-soluble dye, Sudan III (Wako Pure Chemical Industries, Ltd.), was used to identify the oil phase.

\subsection{Preparation of W/O microemulsion}

To prepare the W/O microemulsion, MEL-A was dissolved in an appropriate amount of chloroform, and the stock solution was distributed into test tubes (about $0.25 \mathrm{~g}$ MEL-A each). After that, the solvent was removed by a rotary evaporator. The sample tubes were kept in a vacuum desiccator at room temperature for over one week to allow the complete removal of solvent. Next, $n$-decane was added to the test tubes containing MEL-A to obtain the MEL-A/ $n$-decane mixtures with various MEL-A/n-decane ratios. The mixtures were then vigorously agitated with a vortex mixer and temperature-cycled several times between 25 and $70{ }^{\circ} \mathrm{C}$ until thoroughly mixed. Finally, distilled water (Milli-Q) was added to the MEL-A/n-decane mixture and mixed as described procedure.

\subsection{DLS measurements}

The size of the colloidal structures was measured with a DLS-7000 instrument (Otsuka Electronics Co., Japan) using a He-Ne laser of $632.8 \mathrm{~nm}$ wavelength as a light source at $25^{\circ} \mathrm{C}$. The time-dependent correlation function of the scattered-light intensity was measured at a scattering angle of $20^{\circ}$. The DLS intensity data were processed using the instrument's software to obtain the hydrodynamic diameter, the polydispersity index, and the mass-diffusion coefficient of the samples. The mass-diffusion coefficient $(D)$ was derived from the decay time $\left(\tau_{\mathrm{C}}\right)$ of the intensity autocorrelation function using $D=\left(2 \mathrm{k}_{\mathrm{L}}^{2} \tau_{\mathrm{C}}\right)^{-1}$, in which $\mathrm{k}_{\mathrm{L}}$ is the scattering wave vector. The hydrodynamic mass-diffusion coefficient $D_{0}$ is obtained as the limit of $D$ as $\mathrm{k}_{\mathrm{L}}$ goes to zero. $D_{0}$ is found to obey the Stokes-Einstein relation, $D_{0}=$ $k T / 6 \Pi \eta R_{\mathrm{H}}$, in which $k$ is the Boltzmann constant, $T$ is the absolute temperature, $\eta$ is the viscosity of the solution, and $R_{\mathrm{H}}$ is the hydrodynamic radius. In this study, the autocorrelation function was analyzed by using the cumulant method.

\subsection{FF-EM measurements}

FF-EM was used to determine the size and shape of the colloidal structure. The samples were frozen with liquid nitrogen at $-189^{\circ} \mathrm{C}$. The fracture process was performed with a JFD-9010 instrument (JOEL, Japan) at $-130^{\circ} \mathrm{C}$ and the fractured surface was then replicated by evaporating platinum at an angle of $60^{\circ}$, followed by carbon at an angle of $90^{\circ}$ to strengthen the replica. The replicate was placed on a copper grid (400 mesh) after being washed with water, methanol, and chloroform. It was then examined and photographed by using a JEM-1010 (JOEL, Japan) transmission electron microscope.

\section{RESULTS AND DISCUSSION}

Here we chose $n$-decane $(\mathrm{C} 10)$ as an oil phase since MEL-A has a similar length of two fatty acid chains (C8 C12) as hydrophobic tails. Figure 1 shows the structure of MEL-A. Firstly, we investigated the miscibility of MEL-A and $n$-decane by varying compositions, and it was found that MEL-A and $n$-decane were completely miscible at all compositions at room temperature, indicating that MEL-A is a relatively hydrophobic surfactant.

Then we attempted to prepare $\mathrm{W} / \mathrm{O}$ microemulsion by the addition of water to MEL-A/ $n$-decane mixtures. Interestingly, the samples kept their single-phase transparent appearance even when a large amount of water was intro- 


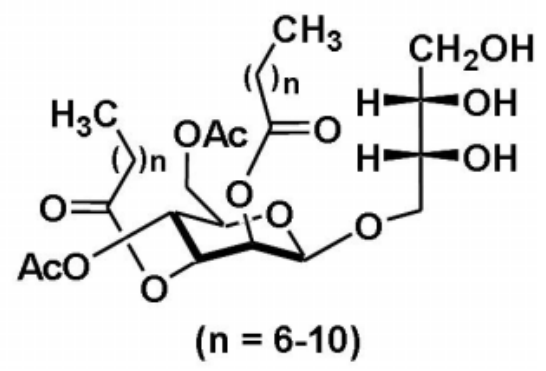

Fig. 1 Structure of MEL-A.

$\mathrm{Ac}=$ acetyl group.

duced beyond its solubility against $n$-decane $(0.0072 \mathrm{wt} \%)^{16)}$, suggesting the formation of a W/O microemulsion. On further addition of water, the samples became turbid indicating the arrival of their solubilization limits.

We then confirmed W/O microemulsion formation in a single phase region using DLS. Figure 2 shows the mean diameter of colloidal structure obtained from the ternary MEL-A/water/ $n$-decane system as a function of water-tosurfactant mole ratio $\left(W_{0}\right)$. From the results, the formation of microemulsions ranging from 20 to $60 \mathrm{~nm}$ was confirmed, which is within the typical range of the microemulsion system $(<100 \mathrm{~nm})^{1-3}$. Moreover, the diameter increased with an increase in $W_{0}$ value (Fig. 2), even though it remained almost constant when $W_{0}$ value was fixed ( $n$-decane to MEL-A molar ratio was increased) as shown in Table 1. This corresponds well to the typical features of W/O microemulsions formed by other surfactants such as $\mathrm{AOT}_{1}{ }^{17-19)}$.

The type of microemulsion was then checked as follows: 1) Small drops of the microemulsion were put into a test tube containing pure water or pure $n$-decane and observed. As a result, the microemulsion dispersed readily in pure $n^{-}$ decane, but still kept the drop-shape in distilled water. 2) The effect of adding an oil-soluble dye (Sudan III) on the visual appearance of the microemulsion was tested. The sample was strongly colored by the oil-soluble dye as shown in Figure 3 indicating that the oil phase was the continuous phase. These lines of evidence strongly indicated the formation of a W/O microemulsion ${ }^{1)}$.

Then, the structure of the W/O microemulsion was directly observed using the FF-EM technique, and Figure 4 shows the freeze fracture electron micrographs of the ternary MEL-A/water $/ n$-decane system $\left(W_{0}=1.5\right.$; Fig. 3a, $W_{0}=8.3$; Fig. $3 \mathrm{~b}$, and $W_{0}=14.3$; Fig. 3c). The images give almost spherical particles whose diameters are nearly equal to those from DLS measurements. The deformed shape of the microemulsion is probably due to a relatively high $W_{0}$ value. $W_{0}$ value at the solubilization limit $\left(W_{\max }\right)$ is an important value to estimate the surfactant's capacity to uptake water in media. Our solubilization limit measurements at various compositions revealed that $W_{\max }$ of MEL-

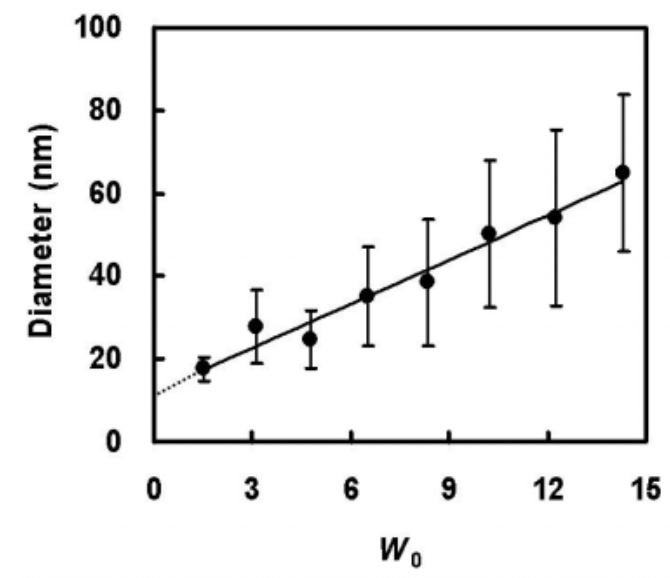

Fig. 2 Mean Diameter of Colloidal Structures Obtained from Ternary MEL-A/water/n-decane System ( $n$ decane/MEL-A molar ratio was 4.8) as a Function of Water-to-surfactant Mole Ratio $\left(W_{0}\right)$ at $25^{\circ} \mathrm{C}$.

Table 1 Mean Diameter of Colloidal Structures Obtained from Ternary MEL-A/water/n-decane System $\left(W_{0}=9.4\right)$ with Different $n$-decane/MEL-A Molar Ratios at $25^{\circ} \mathrm{C}$.

\begin{tabular}{c|c}
\hline$n$-Decane/MEL-A molar ratio & Diameter (nm) \\
\hline 1.5 & $34.4 \pm 10.6$ \\
3.9 & $31.9 \pm 9.1$ \\
8.8 & $32.7 \pm 7.7$ \\
\hline
\end{tabular}

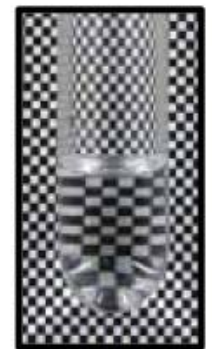

(a)

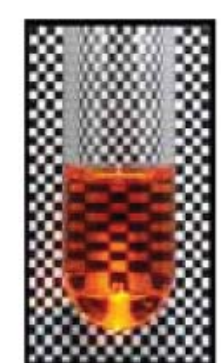

(b)
Fig. 3 The Effect of Adding an Oil-soluble Dye (Sudan III) on the Visual Appearance of the Microemulsion ( $n$-decane/MEL-A molar ratio was 4.8).

(a) microemulsion without dye, (b) microemulsion with dye.

A is 20. Although the value $\left(W_{\max }=20\right)$ is not as high as that of $\operatorname{AOT}\left(W_{\max }=35 \text { for } \mathrm{AOT} / \text { water/decane }\right)^{20)}$, it is almost the same as that of soybean lecithin ${ }^{11)}$. This is the first observation of $\mathrm{W} / \mathrm{O}$ microemulsion formation based on microbial "glycolipid biosurfactant" without using any 


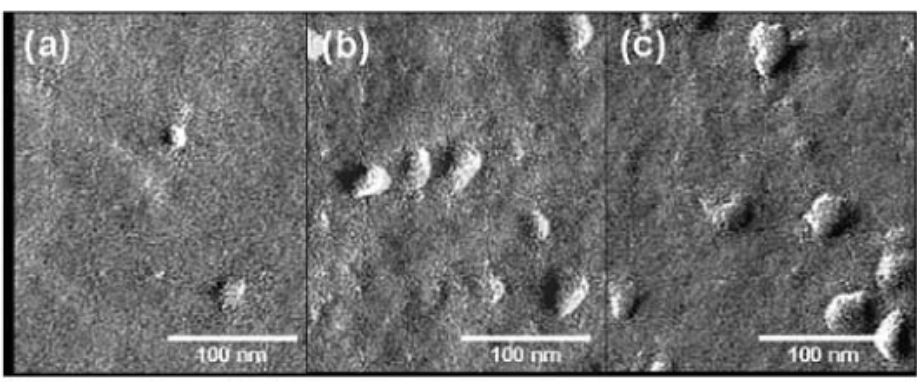

Fig. 4 Freeze Fracture Electron Micrographs of Colloidal Structures Obtained from Ternary MEL-A/water/ $n$ decane System ( $n$-decane / MEL-A molar ratio was 4.8). Bar length is $100 \mathrm{~nm}$. (a) $W_{0}=1.5$, (b) $W_{0}=8.3$, and (c) $W_{0}=14.3$.

cosurfactants.

The possibility of forming a microemulsion strongly depends on the balance between the surfactant's hydrophilic and lipophilic properties, determined not only by the surfactant's chemical structure but also by other factors such as temperature, salinity, and cosurfactants ${ }^{4)}$. Interestingly, glycolipid biosurfactant of MEL-A, which is molecularly engineered by microorganisms, has an excellent combination of hydrophilic and hydrophobic groups for the formation of W/O microemulsion at room temperature without adding any salt or cosurfactants.

Moreover, glycolipid biosurfactant-based microemulsion has a great advantage not only for environmental but also for biological applications including protein crystallization and separation matrix ${ }^{21)}$, or enzymatic reaction media ${ }^{22,23)}$. This is because the sugar moiety of MEL-A was previously found to give an antimicrobial activity as well as a high affinity toward various proteins such as immunoglobulins ${ }^{24)}$ and lectins ${ }^{25}$. Therefore, glycolipid biosurfactant is a useful surfactant for the formation of W/O microemulsion.

\section{CONCLUSION}

The formation of a W/O microemulsion based on the single component of MEL-A, a glycolipid biosurfactant, was confirmed by DLS and FF-EM. The mean diameter of the microemulsion ranged from 20 to $60 \mathrm{~nm}$, and increased with an increase in $W_{0}$ value. The estimated $W_{\max }$ value of MEL-A was 20, which is as high as that of soybean lecithin. Glycolipid biosurfactant-based microemulsion would be useful for environmental applications, especially for microemulsion-based fuels, because biosurfactant itself gives a high environmental compatibility and is also abundantly produced from various oils by a microbial conversion process, which inevitably leads to a high usability of microbial biosurfactant in various oils and fuels media.

\section{ACKNOWLEDGEMENT}

This study was supported by Industrial Technology Research Grant Program in 05A33008c from the New Energy and Industrial Technology Development Organization (NEDO) of Japan.

\section{References}

1. Rosen, M.J. Surfactants and Interfacial Phenomena, Wiley-Interscience. New Jersey. pp. 304, 317, 361 (2004).

2. Langevin, D. Microemulsions - Interfacial aspects. Adv. Colloid Interface Sci. 34, 583-595 (1991).

3. Paul, B.K.; Moulik, S.P. Uses and applications of microemulsions. Curr. Sci. 80, 990-1001 (2001).

4. Garti, N.; Clement, V.; Leser, M.; Aserin, A.; Fanun, M. Sucrose ester microemulsions. J. Mol. Liq. 80, 253-296 (1999).

5. Mele, S.; Murgia, S.; Caboi, F.; Monduzzi, M. Biocompatible lipidic formulations: phase behavior and microstructure. Langmuir 20, 5241-5246 (2004).

6. Neuma de Castro Dantas, T.; da Silva, A.C.; Neto, A.A.D. New microemulsion systems using diesel and vegetable oils. Fuel 80, 75-81 (2001).

7. Palazzo, G. ; Lopez, F. ; Giustini, M. ; Colafemmina, G. ; Ceglie, A. Role of the cosurfactant in the CTAB/ water $/ n$-pentanol $/ n$-hexane water-in-oil microemulsion. 1. pentanol effect on the microstructure. J. Phys. Chem. B 107, 1924-1931 (2003).

8. Larche, F.C.; Delord, P. Structures and stability of isotropic phases in the AOT-decane-water system. Fluid Phase Equilib. 20, 257-264 (1985).

9. Kunieda, H.; Shinoda, K. Solution behavior and hydrophile-lipophile balance temperature in the Aerosol OT-isooctane-brine system: correlation between microemulsions and ultralow interfacial tensions. J. Colloid Interface Sci. 75, 601-606 (1980). 
10. Kotlarchyk, M. ; Chen, S.-H. ; Huang, J.S. ; Kim, M.W. Structure of three-component microemulsions in the critical region determined by small-angle neutron scattering. Phys. Rev. A 29, 2054-2069 (1984).

11. Schurtenberger, P.; Peng, Q.; Leser, M.E.; Luisi, P.-L. Structure and phase behavior of lecithin-based microemulsions: a study of the chain length dependence. J. Colloid Interface Sci. 156, 43-51 (1993).

12. Kitamoto, D.; Isoda, H.; Nakahara, T. Functions and potential applications of glycolipid biosurfactants from energy-saving materials to gene delivery carriers. $J$. Biosci. Bioeng. 94, 187-201 (2002).

13. Imura, T.; Yanagishita, H.; Kitamoto, D. Coacervate formation from natural glycolipid: one acetyl group on the headgroup triggers coacervate-to-vesicle transition. J. Am. Chem. Soc. 126, 10804-10805 (2004).

14. Imura, T.; Ohta, N.; Inoue, K.; Yagi, N.; Negishi, H.; Yanagishita, H.; Kitamoto, D. Naturally engineered glycolipid biosurfactants leading to distinctive self-assembled structures. Chem. Eur. J. 12, 2434-2440 (2006).

15. Imura, T.; Hikosaka, Y.; Worakitkanchanakul, W.; Sakai, H.; Abe, M.; Konishi, M.; Minamikawa, H.; Kitamoto, D. Aqueous-phase behavior of natural glycolipid biosurfactant mannosylerythritol lipid A: sponge, cubic, and lamellar phases. Langmuir 23, 1659-1663 (2007).

16. Schatzberg, P. Solubilities of water in several normal alkanes from $\mathrm{C}_{7}$ to $\mathrm{C}_{16}{ }^{1}$. J. Phys. Chem. 67, 776-779 (1963).

17. Zulauf, M.; Eicke, H.-F. Inverted micelles and microemulsions in the ternary system $\mathrm{H}_{2} \mathrm{O}$ /AerosolOT/isooctane as studied by photon correlation spectroscopy. J. Phys. Chem. 83, 480-486 (1979).

18. Steytler, D.C.; Dowding, P.J.; Robinson, B.H.; Hague, J.D.; Rennie, J.H.S.; Leng, C.A.; Eastoe, J.; Heenan, R.K. Characterization of water-in-oil microemulsions formed in silicone oils. Langmuir 14, 3517-3523 (1998).

19. Ekwall, P.; Mandell, L.; Fontell, K. Some observations on binary and ternary Aerosol OT systems. J. Colloid Interface Sci. 33, 215-235 (1970).

20. Hait, S.K.; Moulik, S.P.; Rodgers, M.P.; Burke, S.E.; Palepu, R. Physicochemical studies on microemulsions. 7. dynamics of percolation and energetics of clustering in water/AOT/isooctane and water/AOT/decane w/o microemulsions in presence of hydrotopes (sodium salicylate, $\alpha$-naphthol, $\beta$-naphthol, resorcinol, catechol, hydroquinone, pyrogallol and urea) and bile salt (sodium cholate). J. Phys. Chem. B 105, 7145-7154 (2001).

21. Vasudevan, M.; Wiencek, J.M. Mechanism of the extraction of proteins into Tween 85 nonionic microemulsions. Ind. Eng. Chem. Res. 35, 1085-1089 (1996).

22. Bauduin, P.; Touraud, D.; Kunz, W.; Savelli, M.-P.; Pulvin, S.; Ninham, B.W. The influence of structure and composition of a reverse SDS microemulsion on enzymatic activities and electrical conductivities. J. Colloid Interface Sci. 292, 244-254 (2005).

23. Holmberg, K. Organic and bioorganic reactions in microemulsions. Adv. Colloid Interface Sci. 51, 137174 (1994).

24. Imura, T.; Ito, S.; Azumi, R.; Yanagishita, H.; Sakai, H.; Abe, M.; Kitamoto, D. Monolayers assembled from a glycolipid biosurfactant from Pseudozyma (Candida) antarctica serve as a high-affinity ligand system for immunoglobulin G and M. Biotechnol. Lett. 29, 865-870 (2007).

25. Konishi, M.; Imura, T.; Fukuoka, T.; Morita, T.; Kitamoto, D. A yeast glycolipid biosurfactant, mannosylerythritol lipid, shows high binding affinity towards lectins on a self-assembled monolayer system. Biotechnol. Lett. 29, 473-480 (2007). 